Bull. Korean Math. Soc. 51 (2014), No. 5, pp. 1281-1289

http://dx.doi.org/10.4134/BKMS.2014.51.5.1281

\title{
ON THE TRANSCENDENTAL ENTIRE SOLUTIONS OF A CLASS OF DIFFERENTIAL EQUATIONS
}

\author{
Weiran Lü, QiUying Li, And Chungchun YANG
}

ABSTRACT. In this paper, we consider the differential equation

$$
F^{\prime}-Q_{1}=R \mathrm{e}^{\alpha}\left(F-Q_{2}\right),
$$

where $Q_{1}$ and $Q_{2}$ are polynomials with $Q_{1} Q_{2} \not \equiv 0, R$ is a rational function and $\alpha$ is an entire function. We consider solutions of the form $F=f^{n}$ where $f$ is an entire function and $n \geq 2$ is an integer, and we prove that if $f$ is a transcendental entire function, then $\frac{Q_{1}}{Q_{2}}$ is a polynomial and $f^{\prime}=\frac{Q_{1}}{n Q_{2}} f$. This theorem improves some known results and answers an open question raised in [16].

\section{Introduction and main results}

In this paper, a meromorphic (resp. entire) function means meromorphic (resp. analytic) in the whole complex plane. We shall adopt the standard notations in Nevanlinna's value distribution theory of meromorphic functions. Such as, the characteristic function

$$
T(r, f)=N(r, f)+m(r, f),
$$

the counting function of the poles

$$
N(r, f)=\int_{0}^{r} \frac{n(t, f)-n(0, f)}{t} d t+n(0, f) \log r,
$$

the proximity function

$$
m(r, f)=\frac{1}{2 \pi} \int_{0}^{2 \pi} \log ^{+}\left|f\left(r e^{i \theta}\right)\right| d \theta,
$$

where

$$
\log ^{+} x=\log \max \{x, 1\}=\max \{\log x, 0\}(x \geq 0),
$$

and the reduced counting function $\bar{N}(r, f)$ (see, e.g., $[6,14]$ ).

Received November 9, 2011; Revised February 6, 2013.

2010 Mathematics Subject Classification. 30D35.

Key words and phrases. transcendental entire solutions, differential equation, Nevanlinna theory. 
Given a meromorphic function $f$, recall that $\alpha \not \equiv 0, \infty$ is a small function with respect to $f$, if $T(r, \alpha)=S(r, f)$, where $S(r, f)$ denotes any quantity satisfying $S(r, f)=o\{T(r, f)\}$ as $r \rightarrow \infty$, possibly outside a set of $r$ of finite linear measure. In addition, let $f$ and $g$ be two nonconstant meromorphic functions, $a \in \mathbb{C} \cup\{\infty\}$. If $f-a$ and $g-a$ have the same zeros with the same multiplicities, we say that they share the value $a \mathrm{CM}$ (Counting multiplies).

The subject on sharing values between entire functions and their derivatives was first studied by Rubel and Yang [12]. They proved a result in 1977 that if a non-constant entire function $f$ and $f^{\prime}$ share two distinct finite numbers $a, b \mathrm{CM}$, then $f=f^{\prime}$. Since then, shared value problems have been studied by many authors and a number of profound results have been obtained (see, e.g., $[5,11,17])$.

To state our main result, we also need the following concepts.

Definition 1. The order $\rho(f)$ and the hyper-order $\rho_{2}(f)$ of a meromorphic function $f$ are defined by

$$
\rho(f)=\limsup _{r \rightarrow \infty} \frac{\log T(r, f)}{\log r}, \quad \rho_{2}(f)=\limsup _{r \rightarrow \infty} \frac{\log \log T(r, f)}{\log r},
$$

respectively.

In 1996, Brück [1] studied the relation between $f$ and $f^{\prime}$ if an entire function $f$ shares only one finite value CM with its derivative $f^{\prime}$. Meanwhile, he posed the following famous conjecture.

Brück Conjecture. Let $f$ be a non-constant entire function such that the hyper-order $\rho_{2}(f)$ of $f$ is not a positive integer and $\rho_{2}(f)<\infty$. If $f$ and $f^{\prime}$ share a finite value a $C M$, then $f^{\prime}-a=c(f-a)$, where $c$ is a nonzero constant.

In fact, Brück Conjecture for the case $a=0$ had been proved by R. Brück in [1]. For the case that $f$ is of finite order had been proved by Gundersen and Yang [5]; the case that $\rho(f)=\infty$ and $\rho_{2}(f)<1 / 2$ had been proved by Chen and Shon [2].

Under some additional assumptions, there are many results related to Brück Conjecture (see, e.g., $[9,10]$ and $[13,15,16]$ ). Based on this, it is interesting to ask whether Brück Conjecture holds if the function $f$ is replaced by $n$-th powers $f^{n}$.

In 2008, C. L. Lei and M. L. Fang et al. proved:

Theorem A ([8]). Suppose that $f$ is a non-constant meromorphic function, $n, k$ are positive integers and $n \geq k+5, a$ is a nonzero constant. If $f^{n}$ and $\left(f^{n}\right)^{(k)}$ share a $C M$, then $f=c \mathrm{e}^{w z / n}$, where $c$ and $w$ are nonzero constants such that $w^{k}=1$.

Recently, J. L. Zhang and L. Z. Yang obtained the following result.

Theorem $\mathbf{B}([15])$. Let $f$ be a non-constant entire function, $n$ be a positive integer. If $f^{n}$ and $\left(f^{n}\right)^{(k)}$ share $1 C M$, and $n \geq k+1$, then $f^{n}=\left(f^{n}\right)^{(k)}$, and $f=c \mathrm{e}^{w z / n}$, where $c$ and $w$ are nonzero constants such that $w^{k}=1$. 
In 2011, F. Lü and H. Yi proved the following theorem by using the theory of normal families.

Theorem C ([10]). Let $f$ be a transcendental entire function, $n, k$ be two integers with $n \geq k+1, F=f^{n}$ and $Q \neq 0$ be a polynomial. If $F-Q$ and $F^{(k)}-Q$ share the value $0 C M$, then $F=F^{(k)}$ and $f=c \mathrm{e}^{w z / n}$, where $c$ and $w$ are nonzero constants such that $w^{k}=1$.

According to the above theorems, one may ask an interesting question: what can be said "if $f^{n}-Q_{1}$ and $\left(f^{n}\right)^{(k)}-Q_{2}$ share the value 0 CM"? where $Q_{1}$ and $Q_{2}$ are polynomials, and $Q_{1} Q_{2} \not \equiv 0$. Our purpose in this article is to solve this question for $k=1$ by giving the transcendental entire solutions of the equation

$$
F^{\prime}-Q_{1}=R \mathrm{e}^{\alpha}\left(F-Q_{2}\right),
$$

where $F=f^{n}, R$ is a rational function and $\alpha$ is an entire function. As an application, we now use a different method from that in $[8,10,16]$ completely and give the following results.

Theorem 1. Let $f$ be a transcendental entire function and let $F=f^{n}$ be $a$ solution of equation (1), $n \geq 2$ be an integer, then $\frac{Q_{1}}{Q_{2}}$ is a polynomial, and

$$
f^{\prime}=\frac{Q_{1}}{n Q_{2}} f
$$

From Theorem 1, we have the following results.

Corollary 1. Let $f$ be a transcendental entire function, $n \geq 2$ be an integer. If $f^{n}-Q$ and $\left(f^{n}\right)^{\prime}-Q$ share $0 C M$, where $Q \not \equiv 0$ is a polynomial, then

$$
f=c \mathrm{e}^{z / n},
$$

where $c$ is a nonzero constant.

Obviously, Corollary 1 shows that Theorem 1 is an improvement of Theorem $\mathrm{C}$ in the case $k=1$, and answers an open question raised in [16].

Corollary 2. If $\frac{Q_{1}}{Q_{2}}$ is not a polynomial, then equation (1) has no transcendental entire solution, where $n \geq 2$ is an integer.

In order to illustrate our condition $n \geq 2$ is sharp, we give examples as follows.

Example 1. Let $f(z)=\mathrm{e}^{\mathrm{e}^{z}} \int_{0}^{z} \mathrm{e}^{-\mathrm{e}^{t}}\left(1-\mathrm{e}^{t}\right) t \mathrm{~d} t$. Then $f$ is a non-constant solution of

$$
f^{\prime}-z=\mathrm{e}^{z}(f-z),
$$

and $f^{\prime}-z, f-z$ share $0 \mathrm{CM}$, while $f \not \equiv f^{\prime}$.

Example 2. Let $f(z)=\mathrm{e}^{3 z}+\frac{2 z}{3}+\frac{2}{9}$. Then $f$ is a non-constant solution of

$$
f^{\prime}-z=3(f-z),
$$

and $f-z, f-z^{\prime}$ share $0 \mathrm{CM}$, but $f \not \equiv f^{\prime}$. 
We give an example to show that the hypothesis of the transcendency of $f$ in Theorem 1 is necessary.

Example 3. Let $f(z)=z, Q_{1}(z)=Q_{2}(z)=2 z^{2}-2 z$, and let $n=2, k=1$. Then $f$ is a solution of

$$
F^{\prime}-Q_{1}=2\left(F-Q_{2}\right)
$$

where $F=f^{2}$, while $f \not \equiv f^{\prime}$.

\section{Preliminaries}

In order to prove the results, we need some lemmas.

Lemma 2.1 ([14, Theorem 1.47]). Let $\alpha$ be a non-constant entire function, and $h=\mathrm{e}^{\alpha}$. Then

$$
T(r, \alpha)=o(T(r, h)), \quad T\left(r, \alpha^{\prime}\right)=S(r, h) .
$$

Lemma 2.2 ([3, 4]). Suppose that $f$ is a transcendental meromorphic function and that

$$
f^{n} P(f)=Q(f)
$$

where $P(f)$ and $Q(f)$ are differential polynomials in $f$ with functions of small proximity related to $f$ as the coefficients and the degree of $Q(f)$ is at most $n$. Then

$$
m(r, P(f))=S(r, f) .
$$

Lemma 2.3 ([7, 14]). Let $f$ be a transcendental meromorphic function. Then

$$
m\left(r, \frac{f^{(l)}}{f}\right)=S(r, f)
$$

for every positive integer $l$.

Lemma 2.4 ([6, 14]). Let $f$ be a meromorphic function, and $\alpha_{i}(i=1,2,3)$ be three distinct small functions of $f$. Then

$$
T(r, f) \leq \bar{N}\left(r, \frac{1}{f-\alpha_{1}}\right)+\bar{N}\left(r, \frac{1}{f-\alpha_{2}}\right)+\bar{N}\left(r, \frac{1}{f-\alpha_{3}}\right)+S(r, f) .
$$

\section{Proof of Theorem 1}

To prove Theorem 1, we first write equation (1) as

$$
\mathrm{e}^{\alpha}=\frac{F^{\prime}-Q_{1}}{R\left(F-Q_{2}\right)}
$$

which and the result of Milloux (see, e.g. [6, Theorem 3.1]) will show that

$$
T\left(r, \mathrm{e}^{\alpha}\right) \leq 3 T(r, F)+O(\log r)+S(r, F)=3 n T(r, f)+S(r, f) .
$$

On combining this with Lemma 2.1, we deduce $T(r, \alpha)=S(r, f)$.

By differentiation to equation (1) we have

$$
F^{\prime \prime}-Q_{1}^{\prime}=\left(R^{\prime}+R \alpha^{\prime}\right) \mathrm{e}^{\alpha}\left(F-Q_{2}\right)+R \mathrm{e}^{\alpha}\left(F^{\prime}-Q_{2}^{\prime}\right) .
$$


Moreover, combining (1) with (2) yields

$$
\left(F^{\prime \prime}-Q_{1}^{\prime}\right)\left(F-Q_{2}\right)=\left(\frac{R^{\prime}}{R}+\alpha^{\prime}\right)\left(F^{\prime}-Q_{1}\right)\left(F-Q_{2}\right)+\left(F^{\prime}-Q_{1}\right)\left(F^{\prime}-Q_{2}^{\prime}\right),
$$

i.e.,

$$
\begin{aligned}
& F^{\prime \prime} F-\left(\frac{R^{\prime}}{R}+\alpha^{\prime}\right) F F^{\prime}-\left(F^{\prime}\right)^{2} \\
= & \left\{Q_{1}^{\prime}-\left(\frac{R^{\prime}}{R}+\alpha^{\prime}\right) Q_{1}\right\} F+Q_{2} F^{\prime \prime}-\left\{\left(\frac{R^{\prime}}{R}+\alpha^{\prime}\right) Q_{2}+Q_{2}^{\prime}+Q_{1}\right\} F^{\prime} \\
& +\left(\frac{R^{\prime}}{R}+\alpha^{\prime}\right) Q_{1} Q_{2}+Q_{1} Q_{2}^{\prime}-Q_{1}^{\prime} Q_{2} .
\end{aligned}
$$

Substituting $F^{\prime}=n f^{n-1} f^{\prime}, F=f^{n}$ into (3) results in

$$
f^{n} f^{n-2} P=Q \text {, }
$$

where

$$
P=n f f^{\prime \prime}-n\left(\frac{R^{\prime}}{R}+\alpha^{\prime}\right) f f^{\prime}-n\left(f^{\prime}\right)^{2},
$$

and $Q$ is a differential polynomial in $f$ with the degree $n$.

Lemma 2.2 gives $m\left(r, f^{n-2} P\right)=S(r, f)$, and

$$
T\left(r, f^{n-2} P\right)=S(r, f) .
$$

We now prove $P \equiv 0$. Suppose that $P \not \equiv 0$, and $n \geq 3$, by (5) and Lemma 2.2, we obtain $T(r, f)=S(r, f)$, which is impossible.

We may now assume that $n=2$. So we get

$$
P=2 f f^{\prime \prime}-2\left(\frac{R^{\prime}}{R}+\alpha^{\prime}\right) f f^{\prime}-2\left(f^{\prime}\right)^{2} .
$$

Since we can now set $n=2$, it follows from (5) and Lemma 2.3 that

$$
T(r, P)=S(r, f), \quad m\left(r, \frac{P}{f^{2}}\right)=S(r, f) .
$$

The two equalities above show that $m\left(r, \frac{1}{f^{2}}\right)=S(r, f)$, and thus

$$
m\left(r, \frac{1}{f}\right)=S(r, f) .
$$

In addition, we can see from the expression of $P$ that the multiple zeros of $f$ must be zeros of $P$, and we get

$$
N\left(r, \frac{1}{f}\right)=\bar{N}\left(r, \frac{1}{f}\right)+S(r, f) .
$$

This together with (7) and the first fundamental theorem of Nevanlinna theory will result in

$$
T(r, f)=\bar{N}\left(r, \frac{1}{f}\right)+S(r, f) .
$$


By (6), we find

(9) $P^{\prime}=2 f f^{\prime \prime \prime}-2\left(\frac{R^{\prime}}{R}+\alpha^{\prime}\right)^{\prime} f f^{\prime}-2\left(\frac{R^{\prime}}{R}+\alpha^{\prime}\right) f f^{\prime \prime}-2\left(\frac{R^{\prime}}{R}+\alpha^{\prime}\right)\left(f^{\prime}\right)^{2}-2 f^{\prime} f^{\prime \prime}$.

Let $z_{0}$ be a simple zero of $f$ such that $R\left(z_{0}\right) \neq 0, \infty$, by (6) and (9), we have $P\left(z_{0}\right)+2\left\{f^{\prime}\left(z_{0}\right)\right\}^{2}=0$ and

$$
P^{\prime}\left(z_{0}\right)=-2\left(\frac{R^{\prime}\left(z_{0}\right)}{R\left(z_{0}\right)}+\alpha^{\prime}\left(z_{0}\right)\right)\left\{f^{\prime}\left(z_{0}\right)\right\}^{2}-2 f^{\prime}\left(z_{0}\right) f^{\prime \prime}\left(z_{0}\right),
$$

which implies that $z_{0}$ is a zero of $P f^{\prime \prime}+\left[\left(\frac{R^{\prime}}{R}+\alpha^{\prime}\right) P-P^{\prime}\right] f^{\prime}$. Let

$$
g=\frac{P f^{\prime \prime}+\left[\left(\frac{R^{\prime}}{R}+\alpha^{\prime}\right) P-P^{\prime}\right] f^{\prime}}{f} .
$$

Obviously $g \not \equiv 0$ and

$$
T(r, g)=S(r, f)
$$

By (10) we obtain

$$
f^{\prime \prime}=\alpha_{1} f+\beta_{1} f^{\prime}
$$

where $\alpha_{1}=g / P, \beta_{1}=P^{\prime} / P-\left(R^{\prime} / R+\alpha^{\prime}\right)$, and

$$
T\left(r, \alpha_{1}\right)=S(r, f), T\left(r, \beta_{1}\right)=S(r, f) .
$$

Substituting (11) into (6) will yield

$$
P=2 \alpha_{1} f^{2}+2\left[\beta_{1}-\left(\frac{R^{\prime}}{R}+\alpha^{\prime}\right)\right] f f^{\prime}-2\left(f^{\prime}\right)^{2} .
$$

Also from (11), we find

$$
f^{\prime \prime \prime}=\alpha_{2} f+\beta_{2} f^{\prime}
$$

where $\alpha_{2}=\alpha_{1}^{\prime}+\alpha_{1} \beta_{1}, \beta_{2}=\alpha_{1}+\beta_{1}^{\prime}+\beta_{1}^{2}$, and

$$
T\left(r, \alpha_{2}\right)=S(r, f), T\left(r, \beta_{2}\right)=S(r, f) .
$$

It follows from (13), (11) and (9) that

$$
P^{\prime}=2 \gamma_{1} f^{2}+2 \gamma_{2} f f^{\prime}-2 \gamma_{3}\left(f^{\prime}\right)^{2},
$$

where

$$
\begin{gathered}
\gamma_{1}=\alpha_{2}-\left(\frac{R^{\prime}}{R}+\alpha^{\prime}\right) \alpha_{1}, \\
\gamma_{2}=\beta_{2}-\left(\frac{R^{\prime}}{R}+\alpha^{\prime}\right) \beta_{1}-\left(\frac{R^{\prime}}{R}+\alpha^{\prime}\right)^{\prime}-\alpha_{1}, \\
\gamma_{3}=\frac{R^{\prime}}{R}+\alpha^{\prime}+\beta_{1} .
\end{gathered}
$$

From (12) and (14), we get

$$
P^{\prime}=2 \gamma_{1} f^{2}+2 \gamma_{2} f f^{\prime}-2 \gamma_{3}\left\{\alpha_{1} f^{2}+\left[\beta_{1}-\left(\frac{R^{\prime}}{R}+\alpha^{\prime}\right)\right] f f^{\prime}-\frac{P}{2}\right\} .
$$


Note $P^{\prime}=\left(\alpha^{\prime}+\beta_{1}+\frac{R^{\prime}}{R}\right) P$, we deduce from (15) that

(16) $\left\{\alpha_{1}^{\prime}-2 \alpha_{1}\left(\alpha^{\prime}+\frac{R^{\prime}}{R}\right)\right\} f+\left\{\beta_{1}^{\prime}-\left(\frac{R^{\prime}}{R}+\alpha^{\prime}\right)^{\prime}-\left(\frac{R^{\prime}}{R}+\alpha^{\prime}\right) \beta_{1}+\left(\frac{R^{\prime}}{R}+\alpha^{\prime}\right)^{2}\right\} f^{\prime}=0$.

Suppose first that $\alpha_{1}^{\prime}-2 \alpha_{1}\left(\frac{R^{\prime}}{R}+\alpha^{\prime}\right) \equiv 0$, then $\alpha_{1}=c R^{2} \mathrm{e}^{2 \alpha}$, where $c$ is a nonzero constant, which implies $T\left(r, \mathrm{e}^{\alpha}\right)=S(r, f)$.

By (1) and (8), we have $Q_{1}-R Q_{2} \mathrm{e}^{\alpha} \equiv 0$, and $f^{\prime}=\frac{Q_{1}}{n Q_{2}} f$, which is the desired result.

We now assume that $\alpha_{1}^{\prime}-2 \alpha_{1}\left(\frac{R^{\prime}}{R}+\alpha^{\prime}\right) \not \equiv 0$, (16) shows that

$$
\bar{N}\left(r, \frac{1}{f}\right)=S(r, f),
$$

which also contradicts with (8). Hence $P \equiv 0$. It follows from (4) and (3) that $Q \equiv 0$ and

$$
F^{\prime \prime} F-\left(\frac{R^{\prime}}{R}+\alpha^{\prime}\right) F^{\prime} F-\left(F^{\prime}\right)^{2}=0 .
$$

In view of (17) this gives

$$
\frac{F^{\prime \prime}}{F^{\prime}}=\frac{R^{\prime}}{R}+\alpha^{\prime}+\frac{F^{\prime}}{F} .
$$

By integration, we have $F^{\prime}=d R F \mathrm{e}^{\alpha}$, where $d$ is a nonzero constant. Substituting this into (1) will yield

$$
(d-1) R f^{n}=\frac{Q_{1}-R Q_{2} \mathrm{e}^{\alpha}}{\mathrm{e}^{\alpha}} .
$$

If $d=1$, by this and equation (1), we have

$$
f^{\prime}=\frac{Q_{1}}{n Q_{2}} f
$$

Now let $d \neq 1$. If $f$ has infinitely many zeros, and if $\mathrm{e}^{\alpha}$ is not constant, then the zeros of $f$ are zeros of $Q_{1}-R Q_{2} \mathrm{e}^{\alpha}$ (up to finitely many which are poles of $R$ ) with multiplicities at least $n$. We have by (18) and Lemma 2.4 that

$$
T\left(r, \mathrm{e}^{\alpha}\right) \leq \bar{N}\left(r, \mathrm{e}^{\alpha}\right)+\bar{N}\left(r, \frac{1}{\mathrm{e}^{\alpha}}\right)+\bar{N}\left(r, \frac{1}{\mathrm{e}^{\alpha}-Q_{1} /\left(R Q_{2}\right)}\right)+S\left(r, \mathrm{e}^{\alpha}\right),
$$

which and $n \geq 2$ may lead to a contradiction, and we know that $\mathrm{e}^{\alpha}$ is a constant, say $\mathrm{e}^{\alpha}=B$, from (18) we get $Q_{1}-B R Q_{2} \equiv 0$, which contradicts the assumption.

Suppose now that $f$ has finitely many zeros, and let $f=m \mathrm{e}^{\beta}$, where $m$ is a polynomial and $\beta$ is an entire function. In this case, (18) can be written as

$$
\frac{(1-d) m^{n}}{Q_{2}} \mathrm{e}^{n \beta}+\frac{Q_{1}}{R Q_{2}} \mathrm{e}^{-\alpha}=1 .
$$

Then from (19), it follows that

$$
n T(r, f)=T\left(r, \mathrm{e}^{n \beta}\right)+S(r, f)=T\left(r, \mathrm{e}^{\alpha}\right)+S(r, f)=S(r, f),
$$


which is impossible. This proves Theorem 1.

We conjecture the following extension of Theorem 1.

Conjecture. Let $f$ be a transcendental entire function, $n$ be a positive integer. If $f^{n}-Q_{1}$ and $\left(f^{n}\right)^{(k)}-Q_{2}$ share $0 C M$, and $n \geq k+1$, then $\left(f^{n}\right)^{(k)}=\frac{Q_{1}}{Q_{2}} f^{n}$. Further, if $Q_{1}=Q_{2}$, then $f=c \mathrm{e}^{w z / n}$, where $Q_{1}, Q_{2}$ are polynomials with $Q_{1} Q_{2} \not \equiv 0$, and $c, w$ are nonzero constants such that $w^{k}=1$.

Finally, we conclude the paper with the following:

Question 1. What can be said if the condition in the Conjecture " $f$ " " be replaced by " $P(f)$ "? where $P(z)=\sum_{i=0}^{n} a_{i} z^{i}$.

Question 2. What can be said if the condition in the Conjecture " $\left(f^{n}\right)^{(k)}$ " be replaced by " $\left\{f\left(z+c_{1}\right) f\left(z+c_{2}\right) \cdots f\left(z+c_{n}\right)\right\}{ }^{(k)}$ "? where $c_{j}(j=1,2, \ldots, n)$ are constants.

Acknowledgement. The authors would like to thank the referee for his/her several important suggestions and for pointing out some errors in our original manuscript. These comments greatly improved the readability of the paper.

This works was supported by the research funds of China University of Petroleum (No.14CX06085A).

\section{References}

[1] R. Brück, On entire functions which share one value CM with their first derivatives, Results Math. 30 (1996), no. 1-2, 21-24.

[2] Z. X. Chen and K. H. Shon, On conjecture of R. Brück concerning the entire function sharing one value CM with its derivative, Taiwanese J. Math. 8 (2004), no. 2, 235-244.

[3] J. Clunie, On integral and meromorphic functions, J. London Math. Soc. 37 (1962), $17-22$.

[4] W. Doeringer, Exceptional value of differential polynomials, Pacific J. Math. 98 (1982), no. $1,55-52$.

[5] G. G. Gundersen and L. Z. Yang, Entire functions that share one value with one or two of their derivatives, J. Math. Anal. Appl. 223 (1998), no. 1, 88-95.

[6] W. K. Hayman, Meromorphic Functions, Clarendon Press, Oxford, 1964.

[7] I. Laine, Nevanlinna Theory and Complex Differential Equations, Walter de Gruyter, Berlin, 1993.

[8] C. L. Lei, M. L. Fang, et al., A note on unicity of meromorophic functions, Acta Math. Sci. Ser. A. Chin. Ed. 28 (2008), no. 5, 802-807.

[9] F. Lü, A note on the Brück conjecture, Bull. Korean Math. Soc. 48 (2011), no. 5, 951-957.

[10] F. Lü and H. X. Yi, The Brück conjecture and entire functions sharing polynomals with their $k$-th derivatives, J. Korean Math. Soc. 48 (2011), no. 3, 499-512.

[11] E. Mues and N. Steinmetz, Meromorphe Funktionen, die mit ihrer Ableitung Werte teilen, Manuscripta Math. 29 (1979), no. 2-4, 195-206.

[12] L. A. Rubel and C. C. Yang, Values shared by an entire function and its derivative, Complex Analysis (Proc. Conf., Univ. Kentucky, Lexington, Ky., 1976), 101-103, Lecture Notes in Math., Vol. 599, Springer, Berlin, 1977.

[13] L. Z. Yang and J. L. Zhang, Non-existence of meromorphic solutions of a Fermat type functional equation, Aequationes Math. 76 (2008), no. 1-2, 140-150. 
[14] H. X. Yi and C. C. Yang, Uniqueness Theory of Meromorphic Functions, Science Press, Beijing, 1995.

[15] J. L. Zhang and L. Z. Yang, A power of an entire function sharing one value with its derivative, Comput. Math. Appl. 60 (2010), no. 7, 2153-2160.

[16] _ A power of a meromorphic function sharing a small function with its derivative, Ann. Acad. Sci. Fenn. Math. 34 (2009), no. 1, 249-260.

[17] T. D. Zhang and W. R. Lü, Notes on a meromorphic function sharing one small function with its derivative, Complex Var. Elliptic Equ. 53 (2008), no. 9, 857-867.

WEIRAN LÜ

Department of Mathematics

China University of Petroleum

Qingdao 266580, P. R. China

E-mail address: luwr@upc.edu.cn

QIUYING LI

Department of Mathematics

China University of Petroleum

Qingdao 266580, P. R. China

E-mail address: lixiaojie211@163.com

ChungChun Yang

Department of Mathematics

China University of Petroleum

QINGDAO 266580, P. R. ChinA

E-mail address: machungchun@gmail.com 